\title{
Influence of Rosemary Extract Addition in Different Phases on the Oxidation of Lutein and WPI in WPI-Stabilized Lutein Emulsions
}

\author{
Duoxia Xu, ${ }^{1}$ Zhanqun Hou, ${ }^{2,3}$ Guorong Liu, ${ }^{1}$ Yanping Cao $\left(\mathbb{D},{ }^{1}\right.$ Atikorn Panya, ${ }^{4}$ Hang Xiao, ${ }^{5}$ \\ and Will Dixon ${ }^{5}$ \\ ${ }^{1}$ Beijing Advanced Innovation Center for Food Nutrition and Human Health (BTBU), School of Food \& Health, Beijing \\ Engineering and Technology Research Center of Food Additives, Beijing Higher Institution Engineering Research Center of Food \\ Additives and Ingredients, Beijing Technology \& Business University, Beijing, China \\ ${ }^{2}$ China National Research Institute of Food and Fermentation Industries Co., Ltd., Beijing 100015, China \\ ${ }^{3}$ Beijing Key Laboratory of the Innovative Development of Functional Staple and the Nutritional Intervention for Chronic \\ Disease, Beijing, China \\ ${ }^{4}$ Food Biotechnology Research Unit, National Center for Genetic Engineering and Biotechnology (BIOTEC), 113 Thailand Science \\ Park, Phaholyothin Rd., Khlong Nueng, Khlong Luang, Pathumthani 12120, Thailand \\ ${ }^{5}$ Department of Food Science, University of Massachusetts, Amherst, MA, USA
}

Correspondence should be addressed to Yanping Cao; caoyp@th.btbu.edu.cn

Received 5 June 2019; Revised 24 September 2019; Accepted 30 September 2019; Published 10 January 2020

Guest Editor: Fuguo Liu

Copyright (c) 2020 Duoxia Xu et al. This is an open access article distributed under the Creative Commons Attribution License, which permits unrestricted use, distribution, and reproduction in any medium, provided the original work is properly cited.

The aim was to investigate rosemary extract with different addition methods affecting the physicochemical stability of WPI-coated lutein emulsions and examine the correlations between lutein degradation and WPI oxidation during storage. First, lutein emulsions containing different concentrations of rosemary extract in the oil phase were prepared. Second, lutein emulsions containing rosemary extract in the oil phase or water phase were studied along with the kinetic reaction of lutein degradation. Moreover, the impact of rosemary extract on the oxidation of WPI and their products was also determined. It was noticed that rosemary extract at $0.05 \mathrm{wt} . \%$ exhibited the best protection of lutein. According to the kinetics analysis of lutein degradation, the direct addition of rosemary extract in the oil phase was more suitable for retarding the degradation of lutein in emulsion than the addition in the aqueous phase due to it being partitioned at the interface. Meanwhile, it was revealed that the addition of rosemary extract in the water phase exhibited better inhibition of the WPI oxidation than addition in the oil phase. The understanding of the association and driving forces of rosemary extract in emulsion systems may be useful for the application of rosemary extract in multicomponent food systems.

\section{Introduction}

Lutein is a yellow pigment with two hydroxyl groups in the conjugated polyene chain presented in fruits and vegetables [1]. It has been reported that lutein has the functional properties of reducing incidences of eye diseases such as agerelated macular degeneration and reducing the risk of cardiovascular diseases, atherosclerosis, and cancer diseases $[2,3]$. However, the conjugated polyene chain of lutein is susceptible to degradation during processing and storage. The degradation of lutein produced a number of initial oxidation products, such as carbon-peroxyl triplet biradicals and epoxides [4]. These reactions can lead to loss of color and bioactivity of lutein in food products.

Dispersing the lipid soluble lutein into the oil phase of oil-in-water emulsions could improve its oxidative stability by emulsifying the oil phase solution with the aqueous phase containing emulsifiers [5]. Some studies have reported the stability of delivery systems enriched with lutein $[6,7]$. The oxidation mechanism of lutein was comprehensively reviewed by Boon et al. [8]. Several factors could influence the degradation of lutein in oil-in-water emulsions including 
size, droplet charge, interface thickness of emulsion droplets, and the addition of antioxidants.

In addition, prooxidants such as transition metals, for example, copper and iron, may lead to electron transfer between lutein and metals by forming lutein radical cation. The formed radical can undergo lutein degradation reactions, leading to lutein loss. In addition, lutein may react with the resulting and already present free radicals by adduct formation reactions or hydrogen abstraction, leading to lutein loss [9]. Therefore, the addition of antioxidant such as transition metal chelators (EDTA) or free radical scavengers (e.g., deferoxamine, $\alpha$-tocopherol, and TBHQ) to emulsions was useful to increase emulsion chemical stability [10].

The use of synthetic antioxidants has become consumers' concern regarding their safety, which promotes the food industry to seek more natural alternatives [11]. Rosemary extracts contain different kinds of components such as rosmarinic acid, carnosic acid, and carnosol that provide one of the major sources of natural antioxidants, which are applied commercially at present in food products [12]. There are studies that showed rosemary extract and rosmarinic and carnosic acids were more effective when added to bulk oil than emulsified corn oil. However, it was also reported that rosmarinic acid probably acted as a prooxidant in emulsions. It was interesting to find that rosemary extracts, carnosic acid, and carnosol efficiently prevented hydroperoxide formation in bulk soybean oil, whereas they exhibited prooxidant activity in emulsions [13]. Lipid oxidation occurrence in emulsions at the surface of oil droplets instead of in the aqueous phase is expected to be the reason for this phenomenon.

Natural antioxidants are particularly difficult to evaluate in emulsions due to the complicated interfacial properties influencing the partition of the antioxidants in multiphase food systems [14]. According to the polar paradox hypothesis, lipophilic compounds are better antioxidants in oil-in-water emulsion, while hydrophilic antioxidants are more effective in the nonpolar system. However, it was reported that some antioxidative compounds did not follow the polar paradox due to the partitioning of antioxidants between the oil and water phases. It was reported that antioxidant activity is dependent on different parameters and that polarity is not the only factor to be taken into account $[15,16]$.

Whey protein is a useful emulsifier and can inhibit the oxidation of dispersed phase by preventing the penetration of prooxidants into the emulsified droplet $[17,18]$. It was found that tryptophan, cysteine, and methionine had the most effective antioxidant activities in proteins. Tryptophan played an important role in antioxidation by allowing oxygen radicals to quench its indolic hydrogen due to serving as a hydrogen donor [19]. Amino acid residues such as tryptophan located at the interface of the oil droplets in emulsions might be preferentially impacted by oxidizing the unsaturated oil phase [20].

It has been a challenging topic for food researchers to understand antioxidant behaviors in complex food systems. Antioxidant could be dynamically distributed between oil and water phases in emulsions. The influence of the addition of rosemary extract in the different phases on the stability of the functional components in emulsion is unclear. Therefore, we evaluated the influence of addition methods (in the oil phase or water phase) of a commercial rosemary extract on the degradation of lutein in oil-in-water emulsions. The kinetic parameter reaction rate constants of process of lutein chemical stability test were presented. Moreover, the impact of rosemary extract on the oxidation of WPI and their products was also determined using spectrofluorometry. The relationship between WPI oxidation and lutein degradation was established. Moreover, the physical stability was evaluated by changes of particle size, zeta-potential, and instability index of lutein emulsions before and after storage. The study focused on developing a delivery system with proper antioxidant addition methods that improved oxidative stability for bioactive compound and protein.

\section{Materials and Methods}

2.1. Materials. Rosemary extract (Shenzhen Hua pure Biotechnology Co., Ltd.) was analyzed to contain $9.01 \%$ carnosol, 9.06\% carnosic acid, 2.16\% rosmarinic acid, $4.02 \%$ oleanolic acid, and $13.3 \%$ ursolic acid. Lutein powder (purity $>97 \%$ ) was obtained from Nanjing Zelang Pharmaceutical Technology Co., Ltd. (Nanjing, China). Whey protein isolate (WPI) was obtained from Jinan SAN Chemical Co., Ltd. (Jinan, China). The manufacturer reported that it contained $97.6 \%$ protein (dry basis). Mediumchain triglyceride (MCT) oil was purchased from Lonza Inc. (Allendale, NJ, USA). Sodium azide was obtained from Sigma-Aldrich (St. Louis, MO). All other reagents were of analytical grade.

2.2. Lutein Emulsion Preparation. WPI was dissolved in the 5.0 $\mathrm{mM}$ phosphate buffer at $\mathrm{pH}$ 7.0. 1.0 M NaOH was added to adjust the $\mathrm{pH}$ of the solution to 7.0 if necessary. The solutions were kept overnight to ensure complete dissolution and dispersion. Sodium azide (0.01 wt.\%) was added in WPI solution to prevent microbial growth.

Lutein emulsion ( $0.5 \mathrm{wt}$ \% WPI) was prepared by adding 5 wt.\% MCT oil containing lutein (0.06 wt.\% in the final emulsion) as the dispersed phase to $95 \mathrm{wt} . \%$ aqueous phase solution at room temperature. The mixture was then prehomogenized using an Ultra-Turrax high-speed blender (B25 model, Shanghai Beierte experimental equipment Co., Ltd.) at a speed of $19000 \mathrm{rpm}$ for $3 \mathrm{~min}$ to form coarse emulsions, which were then passed through a microfluidizer processor (M-110PS model; Microfluidics International Corp., Newton, MA) three times at $50 \mathrm{MPa}$. After that, the $\mathrm{pH}$ was adjusted to 7.0 using $1.0 \mathrm{M} \mathrm{HCl}$ or $\mathrm{NaOH}$. To prepare emulsions for chemical analysis, samples were diluted with $5 \mathrm{mM}$ phosphate buffer solution to a total oil content of $1 \mathrm{wt} . \%$ and then transferred into screw-capped brown bottles flushed with nitrogen.

To study the effect of rosemary extract on the stability of lutein in emulsions, rosemary extract was added to oil phase (MCT) of lutein emulsion at a final concentration of $0,0.02$ wt. $\%, 0.05$ wt. $\%$, and 0.1 wt. $\%$, respectively. To study the 
influence of rosemary extract added in the oil phase and water phases on the oxidation of lutein and WPI in WPIstabilized emulsions, rosemary extract was added to the oil phase and water phase at a final concentration of $0.05 \mathrm{wt} . \%$, respectively.

2.3. Determination of Particle Size. Particle size of WPIcoated lutein emulsions with different concentrations and different addition methods of rosemary extract before and after storage was measured by dynamic light scattering using a Zetasizer Nano-ZS90 (Malvern Instruments, Worcestershire, UK) at a fixed detector angle of $90^{\circ}$. To avoid multiple scattering effects, emulsions were diluted with the same phosphate buffer solution to a final oil droplet concentration of 0.005 wt.\% prior to each measurement. Results were described as cumulants mean diameter (size, $\mathrm{nm}$ ) for droplet size.

2.4. Measurement of Zeta-Potential. The electrical charge (zeta-potential) of WPI-stabilized lutein emulsions with different concentrations and different addition methods of rosemary extract before and after storage was determined using a particle electrophoresis instrument Zetasizer NanoZS90 (Malvern Instruments, Worcestershire, UK). Emulsions were diluted to a droplet concentration of $0.005 \mathrm{wt} . \%$ using buffer solution prior to each measurement to avoid multiple scattering effects. The instrument used the Smoluchowski approximation to obtain the zeta-potential of the droplets.

2.5. Physical Stability Analyzed by LUMiSizer. The physical stability of WPI-stabilized lutein emulsions with different concentrations and different addition methods of rosemary extract before and after storage was measured with the LUMiSizer (LUM GmbH, Berlin, Germany). It employs centrifugal sedimentation to accelerate the occurrence of instability phenomena. The integration graph characterizes the transmitted NIR light as a function of time and position over the entire sample length. The percentage of light absorbance per hour was described as the "instability index." The instrumental parameters used for the measurement were set as follows: volume, $1.8 \mathrm{ml}$ of dispersion; $3000 \mathrm{rpm}$; time $_{\text {Exp }}, 7650 \mathrm{~s}$; time interval, $30 \mathrm{~s}$; and temperature, $25^{\circ} \mathrm{C}$ [21].

2.6. Chemical Stability of Lutein Emulsion. The degradation of lutein emulsion was measured by measuring the change of lutein content in the emulsions during storage in the dark at $55^{\circ} \mathrm{C}$. WPI-coated lutein emulsions with different contents and different addition methods of rosemary extract were transferred into screw-capped brown bottles flushed with nitrogen. The lutein content was measured during the storage at $55^{\circ} \mathrm{C}$ for 7 days. Lutein in emulsion was extracted with ethanol and n-hexane. After that, the absorbance at $440 \mathrm{~nm}$ was measured using a Shimadzu UVmini-1240 UVvis spectrophotometer. The content of lutein during the storage was obtained by referring to a standard curve of lutein prepared under the same condition. The content of lutein in emulsions was calculated as relative lutein $\mathrm{C}$ in percent: $\mathrm{C}_{(t)} / \mathrm{C}_{0}$, where $\mathrm{C}_{(t)}$ was the lutein concentration after storage for a period $t$ and $\mathrm{C}_{0}$ was the lutein concentration before storage [22].

2.7. WPI Oxidation. The oxidation of WPI in lutein emulsion was measured by measuring both the loss of tryptophan fluorescence and the emission of fluorescence by protein oxidation products in emulsions using fluorescence spectroscopy (RF-5301PC, Shimadzu Corp.). Lutein emulsion samples (e.g., without rosemary extract and with the addition of $0.05 \mathrm{wt} . \%$ rosemary extract in the oil phase or water phase) before and after different storage were dispensed in a quartz spectrofluorometer cell. The emission spectra of tryptophan were recorded at $362 \mathrm{~nm}$ with the excitation wavelength established at $283 \mathrm{~nm}$. Emission spectra of WPI oxidation products were recorded at $714 \mathrm{~nm}$ with the excitation wavelength set at $390 \mathrm{~nm} \mathrm{[23].}$

2.8. Statistical Analysis. All emulsions were prepared in duplicate, and all measurements were performed in three times. Data were analyzed by analysis of variance (ANOVA) using the software package SPSS 12.0 for Windows (SPSS Inc., Chicago, IL).

\section{Results and Discussion}

The paper was organized in three different parts. First, the impact of the addition of different concentrations of rosemary extract on the physical and chemical stabilities of lutein emulsions was determined. In the second part, the stability of the lutein emulsions containing rosemary extract in the oil phase or water phase was presented. The degradation of lutein in emulsions was modeled with the first-order kinetic reaction. In the third part, the effect of rosemary extract addition method (in the oil phase or water phase) on the WPI oxidation in emulsion was assessed.

3.1. Impact of Rosemary Extract Concentration on the Physical Stability of Lutein Emulsion. The particle size, zetapotential, and instability index of lutein emulsions were measured as a function of different concentrations of rosemary extract in the oil phase. An understanding of the effect of rosemary extract at different concentrations on the physical property can provide the basis for the chemical stability of lutein emulsions.

The mean particle size was measured before and after the lutein emulsions and 0, $0.02 \mathrm{wt} . \%, 0.05 \mathrm{wt} . \%$, and $0.1 \mathrm{wt} . \%$ rosemary extracts were stored for 7 days at $55^{\circ} \mathrm{C}$, respectively (Figure 1(a)). The droplet size of lutein emulsions without and with different concentrations of rosemary extract did not show any significant differences $(216.8 \pm 3.2,220.1 \pm 4.1$, $220.8 \pm 2.0$, and $216.1 \pm 3.9 \mathrm{~nm}$, respectively). After storage, the mean droplet size of all the four lutein emulsions was slightly increased but did not significantly change during storage (Figure 1(a)), thus indicating that the emulsions 


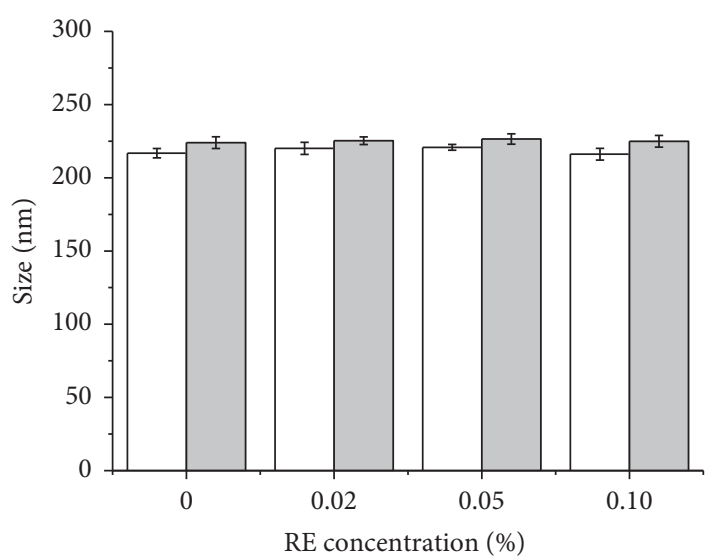

$0 \mathrm{~d}$ $7 d$

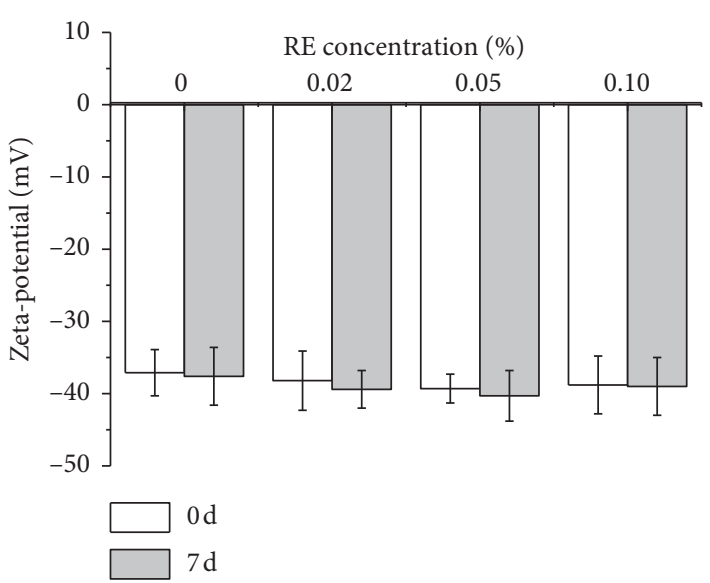

(b)

(a)

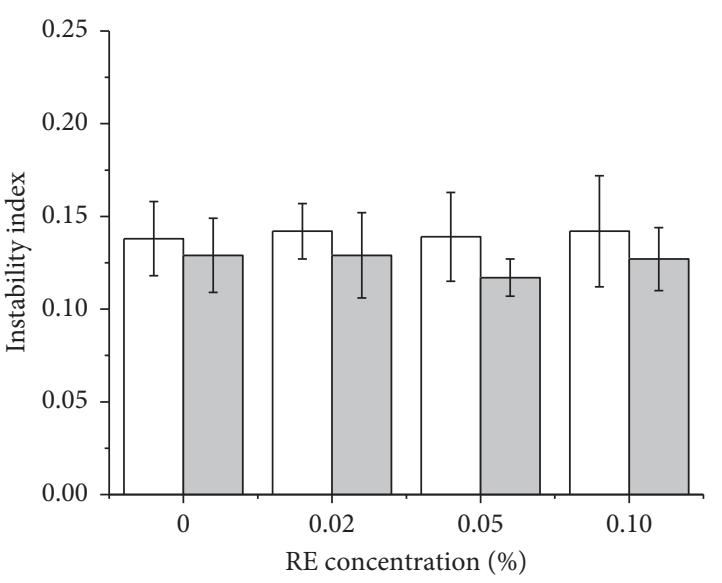

(c)

Figure 1: Effect of the different concentrations of rosemary extract (RE) (in the oil phase) on the droplet size (a), zeta-potential (b), and instability index (c) of WPI-stabilized lutein emulsion before storage ( 0 days) and after storage ( 7 days) at $55^{\circ} \mathrm{C}$. Data points represent means $(n=3) \pm$ standard deviations.

were stable regarding droplet coalescence and Ostwald ripening.

As can be seen in Figure 1(b), there were no significant differences in the zeta-potential of the presence of various concentrations of rosemary extract in lutein emulsions at 0 days compared with the control (the absence of rosemary extract). The droplets of lutein emulsions with $0,0.02 \mathrm{wt} . \%$, 0.05 wt.\%, and 0.1 wt.\% rosemary extract exhibited high negatively charged surfaces ranging from $-37.1 \pm 3.2$ to $-39.3 \pm 1.9 \mathrm{mV}(P>0.05)$, indicating that there was enough electrostatic repulsion between the lutein droplets to prevent them from coming into close proximity.

After 7 days of storage, the zeta-potential of the lutein emulsion droplets with different concentrations of rosemary extract seems to be slightly more negative in comparison with the values at 0 days. However, there were no significant differences in the zeta-potential values in this study $(P>0.05)$.
It was reported that slight decreases in a negatively charged surface of proteins could be attributed to the loss of cationic species such as amines that might react with aldehydes from lutein oxidation [20].

The effect of the addition of rosemary extract on the instability index of lutein emulsion using LUMisizer was demonstrated in Figure 1(c). It was observed that the addition of rosemary extract did not affect the instability index of lutein emulsions. However, thermal treatment during storage slightly decreased the instability indexes. It has been reported that when whey-protein-coated droplets are heated, the layer of adsorbed proteins undergoes conformational changes, leading to exposure of nonpolar and sulfhydryl groups. Thereby, the hydrophobic attraction between the droplets probably was increased [24].

Overall, the results of droplet size, zeta-potential, and instability index proved the insignificant influences of 
different concentrations of rosemary extract on the physical stability of lutein emulsion. Meanwhile, before and after storage, all the four lutein emulsions (without and with different concentrations of rosemary extract) were stable to droplet aggregation.

3.2. Impact of Rosemary Extract Concentration on the Chemical Stability of Lutein Emulsion. Figure 2 shows the impact of rosemary extract concentration on the chemical degradation of lutein in oil-in-water emulsions during storage at $55^{\circ} \mathrm{C}$ accelerated degradation tests. As shown in Figure 2, the protective effect of rosemary extract was observed at the three concentrations (0.02 wt.\%, $0.05 \mathrm{wt} . \%$, and $0.1 \mathrm{wt} . \%$ ) in comparison with the control (without rosemary extract). After storage for $7 \mathrm{~d}$, the lutein emulsion without rosemary extract exhibited a lutein loss of $45.5 \%$, while those with rosemary extract of $0.02 \mathrm{wt} . \%, 0.05 \mathrm{wt} . \%$, and $0.1 \mathrm{wt} . \%$ exhibited lutein losses of $28.3 \%, 12.1 \%$, and $24.1 \%$, respectively. The best chemical stability of lutein emulsion was achieved for the addition of $0.05 \mathrm{wt} . \%$ rosemary extract followed by $0.1 \mathrm{wt} . \%$ and $0.02 \mathrm{wt} . \%$, respectively. More rosemary extracts with concentration of $0.1 \mathrm{wt} . \%$ decreased their effectiveness and even caused prooxidative effect. In addition, it was suggested that phenolic antioxidants become prooxidants by regenerating peroxyl radicals $[25,26]$.

The effective protection of lutein by rosemary extract was due to its composition containing $9.01 \%$ carnosol, $9.06 \%$ carnosic acid, $2.16 \%$ rosmarinic acid, $4.02 \%$ oleanolic acid, and $13.3 \%$ ursolic acid. The effect of adding rosemary extract and its constituents, carnosol, carnosic acid, and rosmarinic acid, in bulk oil and emulsion has been noted by Frankel et al. [13]. It was mentioned that rosemary extract, carnosol, and carnosic acid were effective antioxidants in corn oil emulsions. Rosemary extract was described as an antioxidant that scavenges hydroxyl radicals and peroxyl radicals, preventing lipid peroxidation [26]. Therefore, rosemary extract showed the effective antioxidant properties in the lutein emulsions.

It was also reported that the charge effect of hydrophilic antioxidant ascorbic acid on pigment stability was a function not only of concentrations but also the presence of transition metal ions. According to Qian et al., ascorbic acid could act as a prooxidant attributed to its ability to convert ferric ions into more reactive ferrous ions, which promoted the formation of free radicals [27]. Therefore, it was very useful to understand the effect of rosemary extract content on the degradation of lutein emulsion. At higher concentrations, it might negatively affect lutein stability. In this study, it was shown that the addition of $0.1 \mathrm{wt} . \%$ extract had a less positive effect on lutein stability compared with 0.05 wt.\% extract, which was probably due to high amounts of antioxidants acting counterintuitively as a prooxidant. Panya et al. proved that rosmarinic acid and $\alpha$-tocopherol in the oil phase of the emulsion exhibited strong synergistic interactions in waterin-oil emulsions containing lutein [28]. It was reported that this interaction resulted in the formation of caffeic acid from rosmarinic acid, which would enhance the oxidative stability of the emulsion. A similar interaction between rosemary

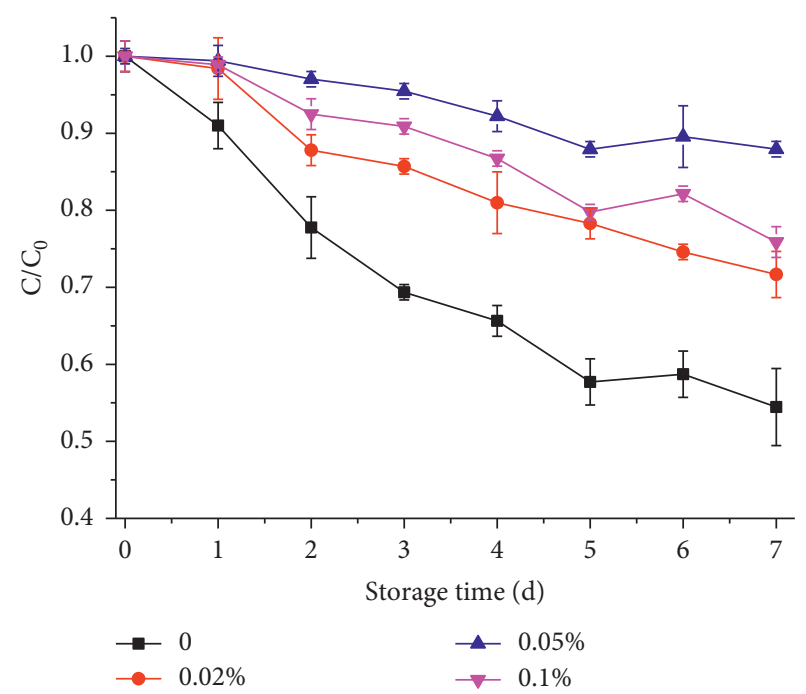

FIGURE 2: Effect of the different concentrations of rosemary extract (in the oil phase) on the degradation of lutein in WPI-stabilized emulsions during the storage at $55^{\circ} \mathrm{C}$. Data points represent means $(n=3) \pm$ standard deviations.

extract and lutein might be occurring in the lutein emulsions studied in this work.

3.3. Influence of the Addition of Rosemary Extract in Different Phases on the Physical Stability of Lutein Emulsion. The droplet size, surface charge, and instability index of lutein emulsions were measured in the present and absence of rosemary extract in both the oil and water phases (Figures 3(a)-3(c)). The droplet size of the lutein emulsions without and with rosemary extract in oil and water phases did not change remarkably after storage at $55^{\circ} \mathrm{C}$ for 7 days, indicating that the three lutein emulsions were relatively physically stable. Before and after storage, zeta-potential was similar for the three lutein emulsions. The slight decrease of zeta-potential after storage could be attributed to the products of lutein oxidation interacting with the amine groups of protein or causing protein confirmation changes [20]. No obvious change of instability index was observed in lutein emulsions without and with rosemary extract in the oil or water phases. There were decreases in the instability index of the three lutein emulsions after storage. It was probably due to the heat induced WPI interaction increasing the interfacial layer thickness and resulted in droplet interactions being dominated by steric hindrance [29]. It indicated that there were no significant differences in the physical stability between the additions of rosemary extract in the oil and water phases.

\subsection{Influence of the Addition of Rosemary Extract in Different} Phases on the Chemical Stability of Lutein Emulsion. The course of the degradation of lutein emulsion with rosemary extract in the oil phase or water phase at $55^{\circ} \mathrm{C}$ was presented in Figure 4. It was found that the addition of rosemary extract in the oil phase or water phase of lutein emulsion inhibited lutein loss during storage. The protection effect of 

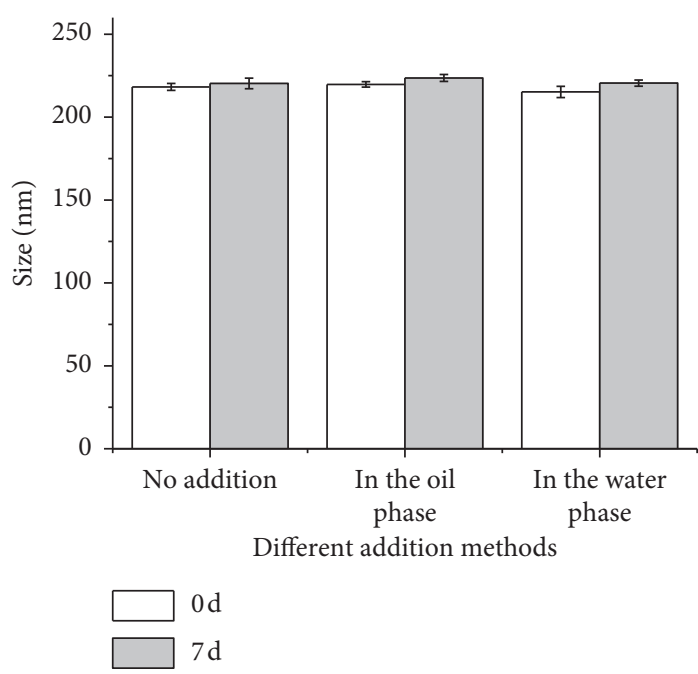
d

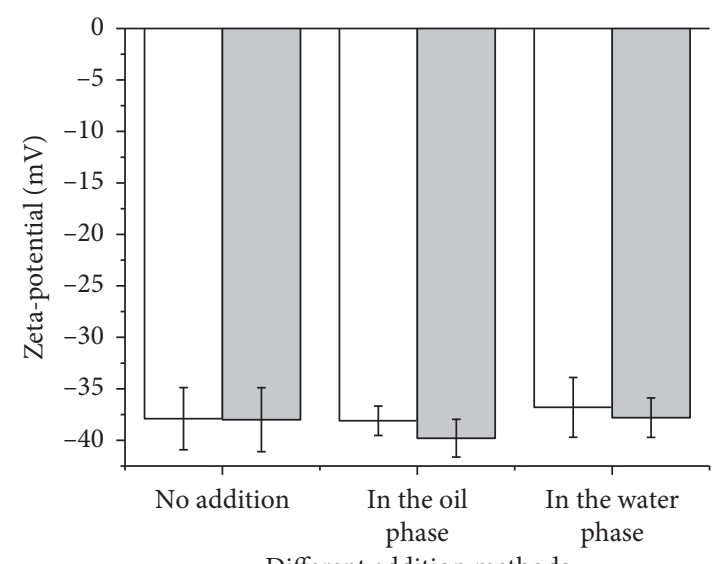

Different addition methods $\square 0 \mathrm{~d}$
$\square 7 \mathrm{~d}$

(a)

(b)

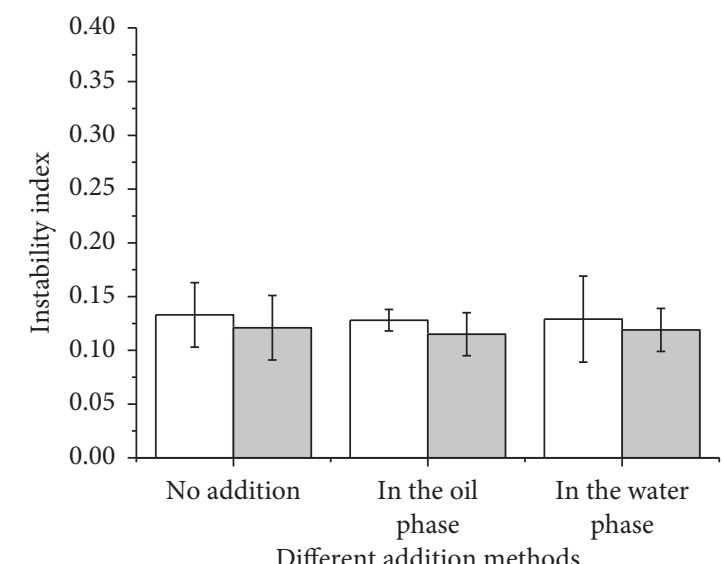

$0 \mathrm{~d}$

$7 d$

(c)

FIGURE 3: Effect of the rosemary extract (RE) addition in different phases (oil and water phase) on the droplet size (a), zeta-potential (b), and instability index (c) of WPI-stabilized lutein emulsion before storage ( 0 days) and after storage ( 7 days) at $55^{\circ} \mathrm{C}$. Data points represent means $(n=3) \pm$ standard deviations.

the addition of rosemary extract in the oil phase of lutein emulsion was much better than that in the water phase. For the emulsion in the absence of rosemary extract, lutein degraded rapidly with $45.5 \%$ loss after 7 -day storage. For the lutein emulsion in the presence of rosemary extract in the oil phase and water phase, lutein degraded with $12.4 \%$ and $25.9 \%$ losses after 7 -day storage, respectively. It proved that the presence of rosemary extract in the oil phase was more suitable for retarding the degradation of lutein in emulsion.

It was found that the first-order kinetic reaction was suitable for the process of lutein loss during the heat chemical stability test. The absolute values of the kinetic parameter reaction rate constant $K$ and the goodness of fit $R^{2}$ were presented in Table 1 . The lower $K$ was, the higher chemical stability of lutein emulsion was. It showed that reaction rate constant $K$ was dependent on the concentration of rosemary extract and the addition methods. The degradation reaction rate constant $K$ of lutein emulsions with different concentrations and different addition phases was in the order of $0.05 \mathrm{wt} . \%$ oil phase $\left(0.81 \times 10^{-3} \cdot \mathrm{h}^{-1}\right)<0.1 \mathrm{wt} . \%$ oil phase $\left(1.57 \times 10^{-3} \cdot \mathrm{h}^{-1}\right)<0.02$ wt. $\%$ oil phase $\left(2.05 \times 10^{-3} \cdot \mathrm{h}^{-1}\right)<0.05$ wt. \% water phase $\left(2.34 \times 10^{-3} \cdot \mathrm{h}^{-1}\right)<$ control $\left(4.04 \times 10^{-3} \cdot \mathrm{h}^{-1}\right)$.

The first-order fitting of lutein degradation was in accordance with other researches [30]. The first-order reaction for chemical stability of lutein emulsion proved that the mechanisms of degradation of carotenoids were similar. It also indicated that the kinetic parameter of carotenoids degradation was dependent on the content and addition phase of antioxidants used. Moreover, the presence of the rosemary extract in the oil or aqueous phase of the lutein emulsions did not change the type of chemical reaction acting on the lutein. 


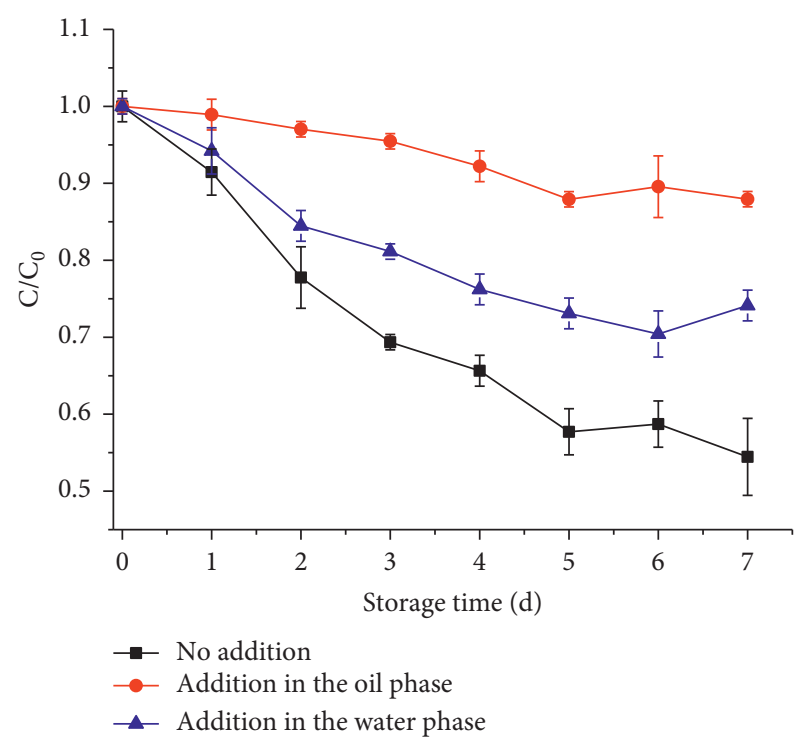

FIGURE 4: Effect of the rosemary extract (RE) addition in different phases (oil and water phase) on the degradation of lutein in WPIstabilized emulsions during the storage at $55^{\circ} \mathrm{C}$. Data points represent means $(n=3) \pm$ standard deviations.

TABLE 1: First-order reaction rate constant for heat degradation of lutein emulsion with different concentrations and addition methods of rosemary extract.

\begin{tabular}{lcc}
\hline $\begin{array}{l}\text { Different concentration and addition } \\
\text { methods of rosemary extract in lutein } \\
\text { emulsion }\end{array}$ & $k\left(\mathrm{~h}^{-1}\right)$ & $R^{2}$ \\
\hline Control & $4.04 \times 10^{-3}$ & 0.9304 \\
0.02 wt. $\%$ in the oil phase & $2.05 \times 10^{-3}$ & 0.9619 \\
0.05 wt.\% in the oil phase & $0.81 \times 10^{-3}$ & 0.9077 \\
0.1 wt.\% in the oil phase & $1.57 \times 10^{-3}$ & 0.9421 \\
0.05 wt.\% in the water phase & $2.34 \times 10^{-3}$ & 0.8796 \\
\hline
\end{tabular}

In the present study, the addition of rosemary extract in the oil phase was significantly more active than in the corresponding water phase $(P<0.05)$. The result can be explained on the basis of the interfacial properties of the antioxidants. It was assumed that, in the emulsion systems used in this study, the polar rosemary extract in the oil phase was more favorably partitioned at the interface than that in the water phase, thus becoming more protective. The trend was in accordance with the reported results exhibiting that the polar hydrophilic antioxidants ascorbic acid and Trolox were much less protective in emulsion than in bulk systems due to polar paradox [13].

3.5. Impact of Rosemary Extract in Different Phases on the Oxidation of WPI. There are some amino acids such as Cys, Trp, and Met from proteins that present antioxidative potentials with both metal-chelating and radical scavenging activities [31]. WPI oxidation in lutein emulsion occurred during the storage. Estévez et al. reported an approach for the analysis of the protein-lipid interaction and protein oxidation by the decrease of natural tryptophan fluorescence and the increase in fluorescence protein oxidation products through fluorescence spectroscopy [32]. The effects of the addition of rosemary extract in the different phases on the oxidation of WPI in lutein emulsions by decrease of tryptophan fluorescence (Figure 5(a)) and the increase in fluorescence of protein oxidation products (Figure 5(b)) were shown.

When excited at $283 \mathrm{~nm}$, tryptophan residue was the only species to emit fluorescence in the range of 300$400 \mathrm{~nm}$. At the beginning, the addition of rosemary extract in the oil phase or water phase in lutein emulsions did not obviously affect the initial level of tryptophan fluorescence compared with the absence of rosemary extract in emulsion. The tryptophan fluorescence decreased in the three lutein emulsions samples during storage for 7 days. During the first 2 days, the loss of tryptophan fluorescence was slight, and the differences of fluorescence intensity between the additions of rosemary extract in the different phase and the control sample were not significant $(P>0.05)$. After the storage for 3 days, the fluorescence intensity of WPI in lutein emulsions was in the order of rosemary extract in the water phase $>$ rosemary extract in the oil phase $>$ control (no rosemary extract). However, there were no significant differences in the fluorescence intensity of WPI between the additions of rosemary extract in the oil phase and aqueous phase in lutein emulsions. It would therefore indicate that the presence of rosemary extract in the aqueous phase and oil phase of lutein emulsions obviously improved the WPI stability. However, there were no significant differences in the protection of WPI between the additions of rosemary extract in the oil phase and aqueous phase in lutein emulsions. It was indicated that the presence of rosemary extract in the different phases of WPI-stabilized lutein emulsions did not significantly influence the oxidation of WPI. This was probably because most of the WPI molecules were adsorbed into the interface and they could interact with rosemary extract in the oil phase (transferring to the interface) and water phase (much better solubility); therefore, the effects of the addition of rosemary extract in the different phases on the WPI oxidation were similar.

The decrease of fluorescence intensity during storage of WPI-coated lutein emulsions was attributed to the oxidative degradation of tryptophan in the presence of free radical formed during lutein oxidation. Transition metal ions also acted as an initiator converting tryptophan into radicals which could react with lutein leading to its degradation [32]. The decrease of tryptophan fluorescence has been used as an indicator of oxidation of casein and BSA in oil-in-water emulsions [33]. The present result was in agreement with our previous research on the influence of WPI oxidation on the degradation of $\beta$-carotene in WPI-coated emulsions. It was found that the tryptophan fluorescence of WPI decreased in $\beta$-carotene emulsion during storage time and there was a relationship between protein oxidation and $\beta$-carotene loss [34].

The influence of addition of rosemary extract in the different phases on the WPI oxidation products was also studied (Figure 5(b)). The formation of WPI oxidation products due to the reaction of its free amino groups in lutein emulsions without and with addition of rosemary extract in the oil phase and water phase was also detected 


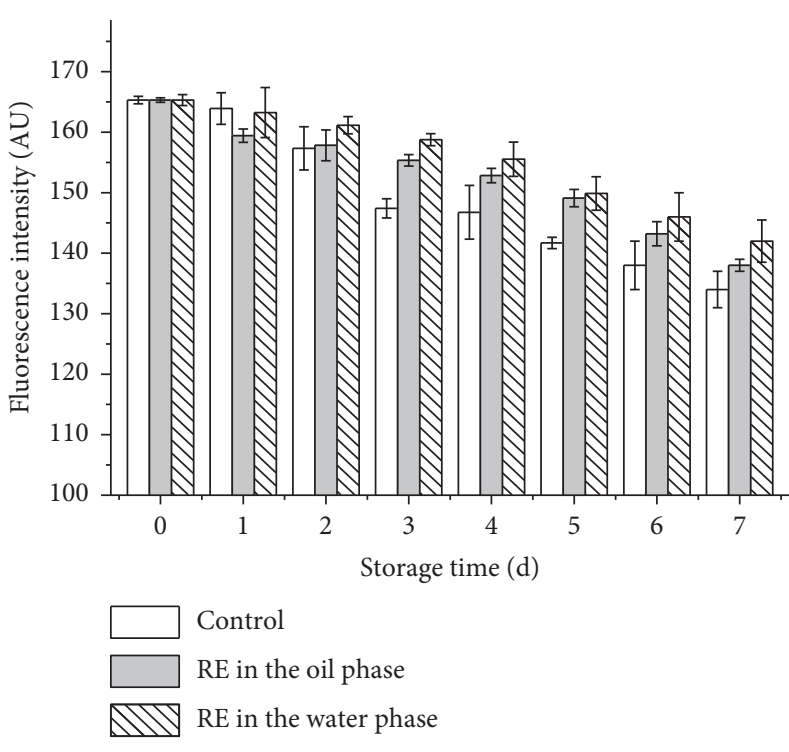

(a)

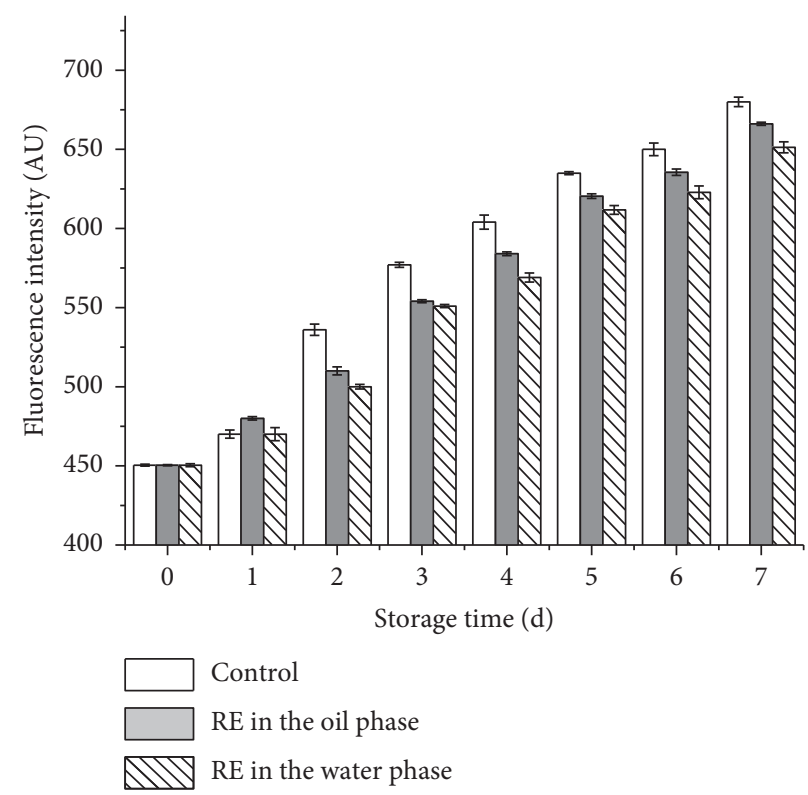

(b)

FIGURE 5: Decrease of tryptophan fluorescence (a) and increase of WPI oxidation products fluorescence (b) in WPI-stabilized lutein emulsions with different addition methods of rosemary extract (RE) (in the oil or water phase) during the storage at $55^{\circ} \mathrm{C}$. Data points represent means $(n=3) \pm$ standard deviations.

using fluorescence spectroscopy. As shown in Figure 5(b), the fluorescence intensity emitted by WPI oxidation products of the three lutein emulsions increased over storage time. Before the storage, the formation of WPI oxidation products was not influenced by the presence of rosemary extract. It was probably due to the fact that the addition of small molecular rosemary extract did not affect the structure of WPI. After storage for 2 days, the addition of rosemary extract in the oil phase and aqueous phase in lutein emulsions obviously decreased the fluorescence intensity compared with the absence of rosemary extract in emulsion. Significant differences of fluorescence intensity were observed between the additions of rosemary extract in the oil phase and aqueous phase in lutein emulsions. The presence of rosemary extract in the water phase significantly decreased the content of WPI oxidation product compared with that in the oil phase during the storage $(P<0.05)$.

It was revealed that the presence of rosemary extract in the aqueous phase of lutein emulsion would be better to inhibit the oxidation of WPI. The correlated change trend of fluorescence intensities of tryptophan and protein oxidation products was expected, since they were generated from WPI oxidation. Lutein emulsion in the absence of rosemary extract exhibited the greatest lutein degradation rate and the highest WPI oxidation product, which was a good correlation between the lutein loss and WPI oxidation. The greater protection of WPI in lutein emulsion with rosemary extract in the water phase might be a function of the better solubility of the rosemary extract mainly containing rosmarinic acid, carnosic, and so forth in the aqueous phase compared with that in the oil phase. Therefore, rosemary extract in the water phase could effectively chelate iron and scavenge free radicals for the WPI oxidation in lutein emulsion. In addition, it was found that rosemary extract in oil phase could provide active prevention against radicals generated from lutein degradation; therefore, the prevention of the radicals generated by WPI might be limited. Finally, the interaction between WPI and rosemary extract could also explain the strong protection of WPI oxidation. It was reported that antioxidant compounds could interact with amino acids [35].

\section{Conclusion}

Overall, the efficacy of rosemary extracts as antioxidants in WPI-stabilized lutein emulsion depends on the concentration and addition method. Rosemary extract was the most active at the certain optimal concentration of $0.05 \mathrm{wt} . \%$. Exceeding it resulted in a decrease of activity and even the occurrence of a prooxidative effect. The first-order reaction kinetic parameter proved that the lutein degradation was dependent on the content and addition phase of antioxidants used. On the protection of lutein in emulsion, rosemary extracts performed better in oil phase than in water phase, whereas, on the protection of WPI, rosemary extracts performed better in water phase than in oil phase. It was assumed that, in the emulsion systems used in this study, the polar rosemary extract in the oil phase was more favorably partitioned at the interface than that in the water phase, thus becoming more protective. The greater protection of WPI in lutein emulsion with rosemary extract in the water phase might be a function of the better solubility of the rosemary extract mainly containing rosmarinic acid, carnosic acid, etc. in the water phase compared with that in the oil phase. In addition, it was found that rosemary extract in oil phase could provide active prevention against radicals generated 
from lutein degradation; therefore, the prevention of the radicals generated by WPI might be limited. As a result, delivery system with rosemary extract can be developed for the emulsification of hydrophobic compounds and also in the protection of protein against oxidation during storage.

\section{Data Availability}

The data used to support the findings of this study are available from the corresponding author upon request.

\section{Conflicts of Interest}

The authors declare that they have no conflicts of interest.

\section{Acknowledgments}

This research was funded by the National Natural Science Foundation of China (31771976), 13th Five-Year Plan of the State Key Development Program (2016YFD0400802), Beijing Key Laboratory of the Innovative Development of Functional Staple and the Nutritional Intervention for Chronic Disease, Beijing Science and Technology Commission (Z171100001317004), Support Project of High-level Teachers in Beijing Municipal Universities in the Period of 13th Five-year Plan (CIT \& TCD201804018), Construction of Service Capability of Scientific and Technological Innovation (PXM2018_014213_000041, PXM2018_014213_000014, and PXM2018_014213_000033), Cultivation and Development of Innovation Base (Z171100002217019), Support Project of High-level Teachers in Beijing Municipal Universities (IDHT20180506), and Quality Construction of Talents Training-First-class Specialty Construction (Municipal Level)Food Science and Engineering (PXM2019_014213_000010). The authors acknowledge Shenzhen Hua pure Biotechnology Co., Ltd for the supply of rosemary extracts.

\section{References}

[1] H. B. Sowbhagya, S. R. Sampathu, and N. Krishnamurthy, "Natural colorant from marigold-chemistry and technology," Food Reviews International, vol. 20, no. 1, pp. 33-50, 2004.

[2] A. Alves-Rodrigues and A. Shao, "The science behind lutein," Toxicology Letters, vol. 150, no. 1, pp. 57-83, 2004.

[3] K. Mitri, R. Shegokar, S. Gohla, C. Anselmi, and R. H. Müller, "Lute in nanocrystals as antioxidant formulation for oral and dermal delivery," International Journal of Pharmaceutics, vol. 420, no. 1, pp. 141-146, 2011.

[4] X. Mesnier, C. Gregory, P. Fança-Berthon, F. Boukobza, and A. Bily, "Heat and light colour stability of beverages coloured with a naturalcarotene emulsion: effect of synthetic versus natural watersoluble antioxidants," Food Research International, vol. 65, pp. 149-155, 2014.

[5] D. J. McClements, E. A. Decker, and J. Weiss, "Emulsion based delivery systems for lipohilic bioactive components," Journal of Food Science, vol. 72, no. 8, pp. R109-R124, 2007.

[6] J. Losso, A. Khachatryan, M. Ogawa, J. Godber, and F. Shih, "Random centroid optimization of phosphatidyl glycerol stabilized lutein-enriched oil-in-water emulsions at acidic pH," Food Chemistry, vol. 92, no. 4, pp. 737-744, 2005.
[7] C. Zhao, H. Cheng, P. Jiang, Y. Yao, and J. Han, "Preparation of lutein-loaded particles for improving solubility and stability by polyvinyl pyrrolidone (PVP) as an emulsion-stabilizer," Food Chemistry, vol. 156, pp. 123-128, 2014.

[8] C. S. Boon, D. J. McClements, J. Weiss, and E. A. Decker, "Factors influencing the chemical stability of carotenoids in foods," Critical Reviews in Food Science and Nutrition, vol. 50, no. 6, pp. 515-532, 2010.

[9] L. B. Fomuso, M. Corredig, and C. C. Akoh, "Metal-catalyzed oxidation of a structured lipid model emulsion," Journal of Agricultural and Food Chemistry, vol. 50, no. 24, pp. 7114-7119, 2002.

[10] D. X. Xu, F. Yuan, Y. X. Gao, D. J. McClements, and E. A. Decker, "Influence of $\mathrm{pH}$, metal chelator, free radical scavenger and interfacial characteristics on the oxidative stability of $\beta$-carotene in conjugated whey protein-pectin stabilised emulsion," Food Chemistry, vol. 139, no. 1-4, pp. 1098-1104, 2013.

[11] A. A. M. Botterweck, H. Verhagen, R. A. Goldbohm, J. Kleinjans, and P. A. Van Den Brandt, "Intake of butylated hydroxyanisole and butylated hydroxytoluene and stomach cancer risk: results from analyses in the Netherlands cohort study," Food and Chemical Toxicology, vol. 38, no. 7, pp. 599-605, 2000.

[12] L. S. Dias, M. E. C. Menis, and N. Jorge, "Effect of rosemary (Rosmarinus officinalis) extracts on the oxidative stability and sensory acceptability of soybean oil," Journal of the Science of Food and Agriculture, vol. 95, no. 10, pp. 2021-2027, 2015.

[13] E. N. Frankel, S.-W. Huang, R. Aeschbach, and E. Prior, "Antioxidant activity of a rosemary extract and its constituents, carnosic acid, carnosol, and rosmarinicacid, in bulk oil and oil-in-water emulsion," Journal of Agricultural and Food Chemistry, vol. 44, no. 1, pp. 131-135, 1996.

[14] M. S. Brewer, "Natural antioxidants: sources, compounds, mechanisms of action, and potential applications," Comprehensive Reviews in Food Science and Food Safety, vol. 10, no. 4, pp. 221-247, 2011.

[15] J. J. Sun, J. H. Liu, and Z. W. Wang, "Application of tea polyphenols to edible oil as antioxidant by W/O microemulsion," Journal of Dispersion Science and Technology, vol. 36, no. 11, pp. 1539-1547, 2015.

[16] M. H. Gordon, F. Paiva-Martins, and M. Almeida, "Antioxidant activity of hydroxytyrosol acetate compared with that of other olive oil polyphenols," Journal of Agricultural and Food Chemistry, vol. 49, no. 5, pp. 2480-2485, 2001.

[17] E. Dickinson, "Hydrocolloids as emulsifiers and emulsion stabilizers," Food Hydrocolloids, vol. 23, no. 6, pp. 1473-1482, 2009.

[18] K. Viljanen, A. L. Halmos, A. Sinclair, and M. Heinonen, "Effect of blackberryand raspberry juice on whey protein emulsion stability," European Food Research and Technology, vol. 221, no. 5, pp. 602-609, 2005.

[19] B. Hernández-Ledesma, A. Dávalos, B. Bartolomé, and L. Amigo, "Preparation of antioxidant enzymatic hydrolysates from (alpha-lactalbumin and beta-lactoglobulin. Identification of active peptides by HPLC-MS/MS," Journal of Agricultural and Food Chemistry, vol. 53, no. 3, pp. 588-593, 2005.

[20] M. S. Katsuda, D. J. McClements, L. H. S. Miglioranza, and E. A. Decker, "Physical and oxidative stability of fish oil-inwater emulsions stabilized with beta-lactoglobulin and pectin," Journal of Agricultural and Food Chemistry, vol. 56, no. 14, pp. 5926-5931, 2008.

[21] D. Xu, X. Wang, J. Jiang, F. Yuan, and Y. Gao, "Impact of whey protein-beet pectin conjugation on the physicochemical stability of beta-carotene emulsions," Food Hydrocolloids, vol. 28, no. 2, pp. 258-266, 2012. 
[22] D. Xu, Z. Aihemaiti, Y. Cao, C. Teng, and X. Li, "Physicochemical stability, microrheological properties and microstructure of lutein emulsions stabilized by multilayer membranes consisting of whey protein isolate, flaxseed gum and chitosan," Food Chemistry, vol. 202, no. 1, pp. 156-164, 2016.

[23] M. Heinonen, D. Rein, M. T. Satué-Gracia, S.-W. Huang, J. B. German, and E. N. Frankel, "Effect of protein on the antioxidant activity of phenolic compounds in a lecithin-liposome oxidation system," Journal of Agricultural and Food Chemistry, vol. 46, no. 3, pp. 917-922, 1998.

[24] K. Demetriades, J. N. Coupland, and D. J. McClements, "Physicochemical properties of whey protein-stabilized emulsions as affected by heating and ionic strength," Journal of Food Science, vol. 62, no. 3, pp. 462-467, 1997.

[25] E. N. Frankel, S.-W. Huang, J. Kanner, and J. B. German, "Interfacial phenomena in the evaluation of antioxidants: bulk oils vs emulsions," Journal of Agricultural and Food Chemistry, vol. 42, no. 5, pp. 1054-1059, 1994.

[26] Y. Che Man and I. Jaswie, "Effect of rosemary and sage extracts on frying performance of refined, bleached and deodorized (RBD) palm olein during deep-fat frying," Food Chemistry, vol. 69, no. 3, pp. 301-307, 2000.

[27] C. Qian, E. A. Decker, H. Xiao, and D. J. McClements, "Nanoemulsion delivery systems: influence of carrier oil on $\beta$-carotene bioaccessibility," Food Chemistry, vol. 135, no. 3, pp. 1036-1043, 2012.

[28] A. Panya, K. Kittipongpittaya, M. Laguerre et al., "Interactions between alpha-tocopherol and rosmarinicacid and its alkyl esters in emulsions: synergistic, additive, or antagonistic effect," Journal of Agricultural and Food Chemistry, vol. 60, no. 41, pp. 10320-10330, 2012.

[29] D. J. McClements, E. A. Decker, Y. Park, and J. Weiss, "Structural design principles for delivery of bioactive components in nutraceuticals and functional foods," Critical Reviews in Food Science and Nutrition, vol. 49, no. 6, pp. 577-606, 2009.

[30] V. Lavelli and M. C. Torresani, "Modelling the stability of lycopene-rich by-products of tomato processing," Food Chemistry, vol. 125, no. 2, pp. 529-535, 2011.

[31] A. Saiga, S. Tanabe, and T. Nishimura, "Antioxidant activity of peptides obtained from porcine myofibrillar proteins by protease treatment," Journal of Agricultural and Food Chemistry, vol. 51, no. 12, pp. 3661-3667, 2003.

[32] M. Estévez, P. Kylli, E. Puolanne, R. Kivikari, and M. Heinonen, "Fluorescence spectroscopy as a novel approach for the assessment of myofibrillar protein oxidation in oil-in-water emulsions," Meat Science, vol. 80, no. 4, pp. 1290-1296, 2008.

[33] K. Viljanen, P. Kylli, R. Kivikari, and M. Heinonen, "Inhibition of protein and lipid oxidation in liposomes by berry phenolics," Journal of Agricultural and Food Chemistry, vol. 52, no. 24, pp. 7419-7424, 2004.

[34] D. Xu, X. Wang, J. Jiang, F. Yuan, E. A. Decker, and Y. Gao, "Influence of $\mathrm{pH}, \mathrm{EDTA}, \alpha$-tocopherol, and WPI oxidation on the degradation of $\beta$-carotene in WPI-stabilized oil-in-water emulsions," LWT-Food Science and Technology, vol. 54, no. 1, pp. 236-241, 2013.

[35] A. Panya, W. Temthawee, N. Phonsatta et al., "Apolarradical initiated conjugated autoxidizabletriene (ApoCAT) assay: effects of oxidant locations on antioxidant capacities and interactions," Journal of Agricultural and Food Chemistry, vol. 63, no. 34, pp. 7546-7555, 2015. 


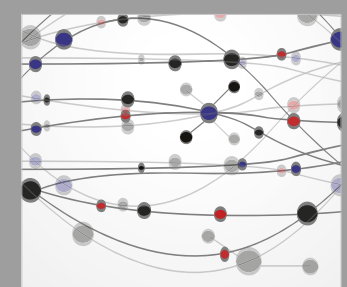

The Scientific World Journal
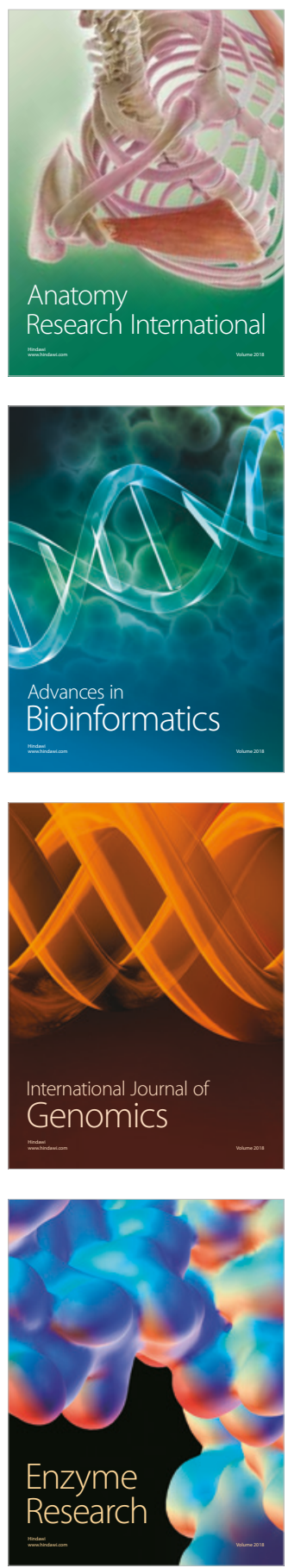
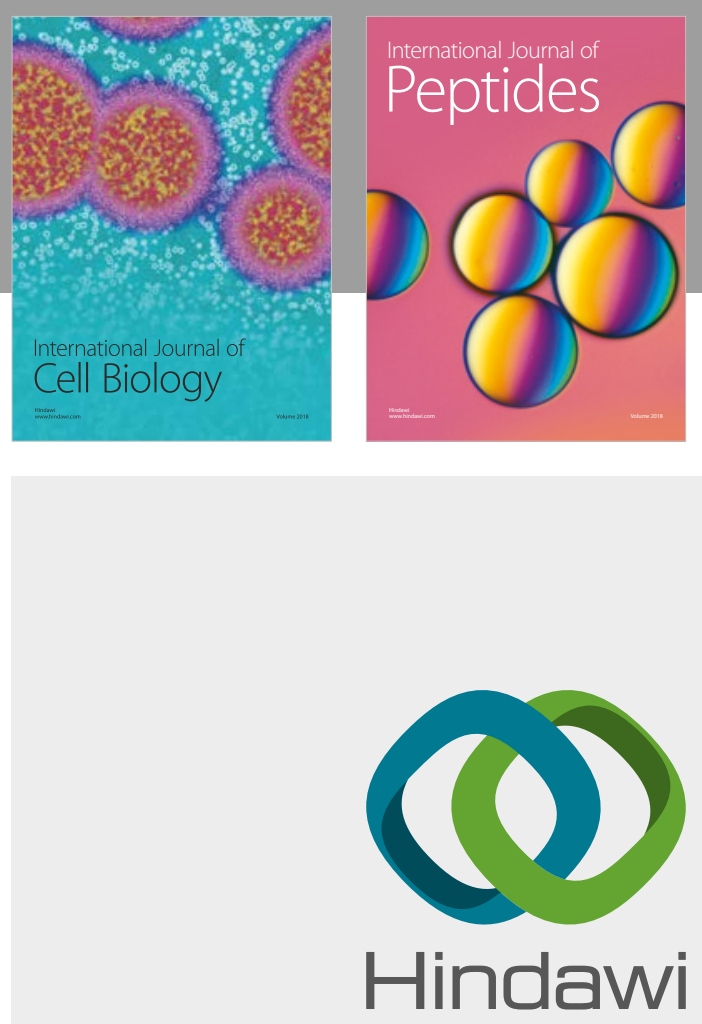

Submit your manuscripts at

www.hindawi.com
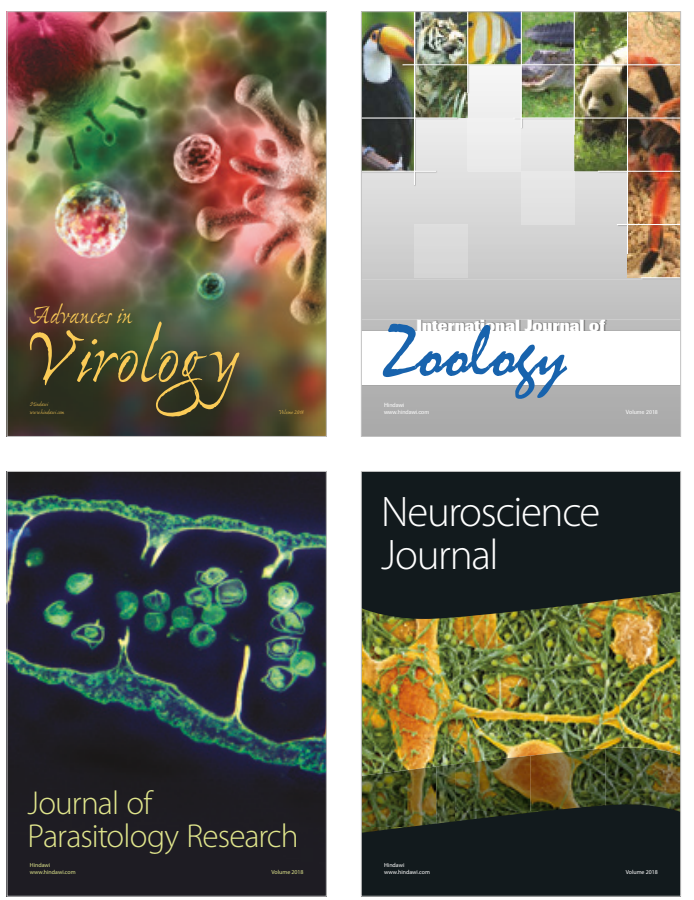
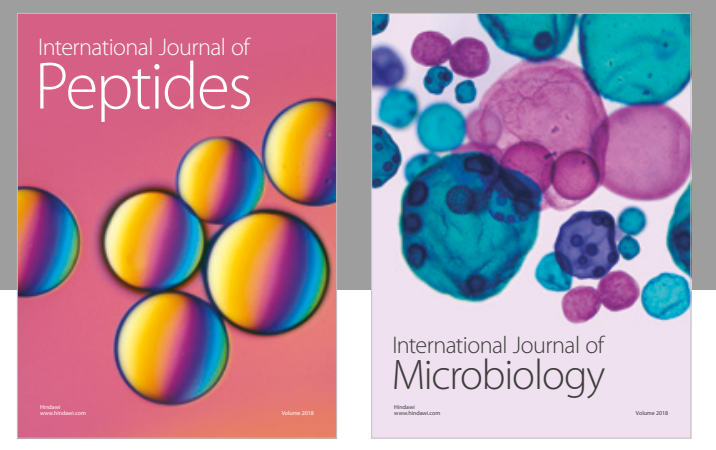

nternational Journal of Microbiology
Journal of
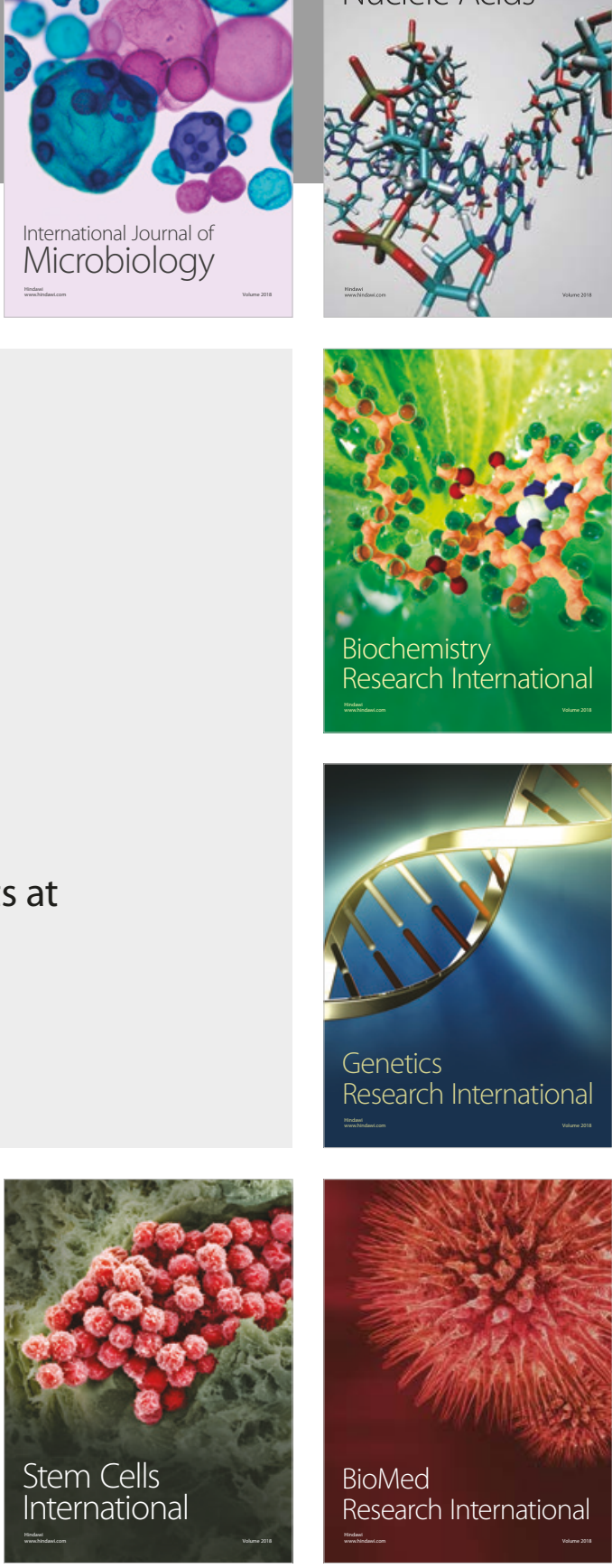
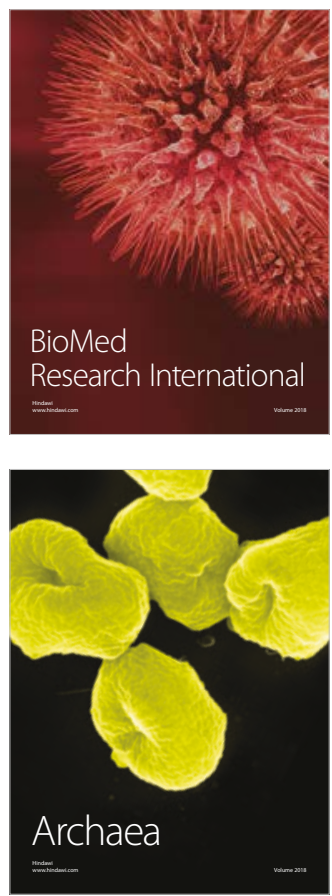